\title{
LINEAR AND CIRCULAR POLARIZATION IN THE DIFFUSE INTERSTELLAR MEDIUM
}

\author{
P. G. MARTIN \\ Canadian Institute for Theoretical Astrophysics
}

\begin{abstract}
A brief introduction is provided to the origin of interstellar polarization, grain model calculations, and alignment. The characteristics of the observed linear and circular continuum polarization are described, with an emphasis on various constraints that might be placed on the nature of the polarizing grains and what additional observations would be of value. Complementary information can be obtained from polarization structure at spectral features in the extinction curve. Interstellar polarization has been measured in some other galaxies, indicating an interesting frontier for further research.
\end{abstract}

\section{INTRODUCTION}

Interstellar polarization of starlight, induced on transmission through a medium of aligned, non-spherical interstellar grains, already provides some unique insights into the physical properties of the dust. Throughout this contribution I point out a number of areas for additional research which to me appear promising. In $\S 2$, I begin with an overview of the production and description of the interstellar polarization, how this can be related to electromagnetic scattering calculations for single grains, and alignment. Next are discussed some inferences that might be drawn from continuum linear polarization ( $(3)$, with some emphasis on the near-infrared and a digression into molecular clouds. The importance of circular polarization is assessed in $\S 4$. Polarization across spectral features from the infrared (again in molecular clouds) to the ultraviolet offers additional insights, as described in $\S 5$. Interstellar polarization in other galaxies is of considerable interest, though presently a relatively undeveloped area of investigation ( $(6)$. Further information might be gleaned from polarization produced by scattering (Martin, 1978), e.g. in reflection nebulae, but this is not discussed here. Hildebrand presented an invited paper on the polarization of far-infrared emission from aligned grains (Hildebrand, 1989). For conciseness I normally cite only relatively recent literature, which ought to be consulted to trace earlier developments.

\section{ORIGIN OF INTERSTELLAR POLARIZATION}

At least some of the interstellar grains are non-spherical and systematically aligned so that the average grain profile projected on the plane of the sky is elongated (the actual particles might be elongated but as discussed below are more likely 
flattened). Radiation propagating through this medium suffers an extinction and phase shift whose magnitude depends on the orientation of the electric vector $(E-$ vector) relative to this grain profile; the medium is said to be linearly dichroic and linearly birefringent, respectively. For typical grain sizes, the differential extinction at optical wavelengths results in linearly polarized starlight whose $E$-vector is oriented along the short axis of the grain profile. An $E$-vector orientation is usually described by a position angle, which in the simplest case for interstellar polarization is constant with wavelength (an important diagnostic not elaborated upon here, but not to be overlooked). The birefringence changes sign, empirically ( $\S 4$ ), at a wavelength $\left(\lambda_{c}\right)$ near that at which the linear polarization is maximum $\left(\lambda_{\max }\right)$. Circular polarization is produced when linearly polarized light is transmitted through the birefringent medium, by the process of linear to circular conversion familiar in a quarter-wave plate, as long as the $E$-vector of the incident radiation is not oriented along either principal axis of the grain profile; thus a change in the position angle of grain alignment along the line of sight to a star (or suitably oriented intrinsically linearly polarized radiation) is required.

Transfer equations for the normalized Stokes parameters that are used to describe the degree of polarization are given by Martin (1974), including the case of large fractional polarization (equation A13 in Martin, 1974; see also Lee and Draine, 1985). Some attention has to be paid to sign conventions for quantities like phases if complex quantities vary as $\exp (-i \omega t)$ as in Lee and Draine (1985) and Draine (1988), and to how the sense of circular polarization is described (Gehrels, 1974a).

The connection to extinction and phase lag cross-sections for single particles can also be seen in Martin (1974) [note a factor two re-definition of the phase lag cross-section in Lee and Draine (1985) and Draine (1988)]. Evaluation of the cross-sections for individual particles can be carried out by various electromagnetic scattering calculations (Bohren and Huffman, 1983). For particles small relative to the wavelength (often relevant in the infrared), analytical expressions for coated confocal spheroids are available (Gilra, 1972; Draine and Lee, 1984). For finite-sized particles, more elaborate calculations are possible for coated confocal spheroids (Onaka, 1980) or coated concentric circular cylinders (Shah, 1970). Calculations for uncoated (homogeneous) particles are more straightforward. More complex shapes and inhomogeneities can be tackled with the 'discrete-dipole method' (Draine, 1988).

The wavelength dependence of the polarization depends on the composition of the grains (through the complex refractive index $m=n-i k$ or the complex dielectric function $\epsilon=m^{2}$ ) and on the distributions of size, coatings, shape, and orientation. Consequently, one might hope to derive some information about these factors from observations; some illustrations are provided below.

The spinning interstellar grains appear to be aligned such that the short axis of the grain profile, and hence the $E$-vector, lies in the direction of the interstellar magnetic field ( $E$-vectors can thus map out the transverse component of the field, an important role of interstellar polarization not discussed here). This alignment is probably produced by magnetic dissipation within the grain which systematically decreases the angular momentum perpendicular to the field direction. Details of the mechanism are less certain. Empirically I am attracted to the view that alignment must not be a marginal process, as it occurs in diverse environments within the Galaxy, and in several others $(\S 6)$. With efficient alignment, polarization to extinction ratios could still be limited to the maximum observed by having only a 
fraction of the grains aligned or by having the grains more spherical (grain axial ratios can in principle be constrained this way, and need not be extreme).

Recent discussions of alignment have been presented by Seki and Hasegawa (1986) and Hildebrand (1988). Davis-Greenstein alignment can be 'improved' by accounting for the alignment of the angular momentum with the grain axis by intern al friction, particularly for oblate particles which realize their potential to be more efficient polarizers than prolate particles, but is probably still not sufficient with ordinary paramagnetic relaxation. Superparamagnetic relaxation could readily produce polarization to extinction ratios higher than observed. Perhaps by default, this idea appears to have a growing following. Whether it is the correct explanation might be checked by searching for regions of 'reverse' alignment predicted if the gas temperature falls below the dust temperature, and for polarization predicted to be absent in regions where the temperatures are equal (can the observed polarization in molecular clouds be explained?). Suprathermal rotation or 'pinwheeling', possibly also benefitting from faster superparamagnetic dissipation, would also improve the alignment, but might be less likely to be activated in molecular clouds. At this symposium, aspects of alignment were discussed in invited papers by Aitken, 1989 and by Hildebrand, 1989 and in contributed papers by Cugnon, 1989 and by Matsumura and Seki, 1989.

\section{OPTICAL AND INFRARED CONTINUUM LINEAR POLARIZATION}

The wavelength dependence of linear polarization can be described by the normalized form

$$
p(\lambda) / p_{\max }=\exp \left[-K \ln ^{2}\left(\lambda_{\max } / \lambda\right)\right]
$$

where $p_{\max }$ is the maximum polarization. This form was proposed originally by Serkowski to describe optical data. Very limited data in the ultraviolet (Gehrels, 1974b) are not inconsistent with this form, and it has been adopted for the extended range $0.22-2.2 \mu \mathrm{m}$ by Wilking, Lebofsky and Rieke (1982), with emphasis on more abundant near-infrared data. They find $K=-0.10+1.86 \lambda_{\max }$, which reproduces the observed progressive narrowing of $p(\lambda)$ as $\lambda_{\text {max }}$ increases (see smooth curves in Fig. 1 for $\lambda_{\max }=0.85,0.7,0.55$, and $\left.0.4 \mu \mathrm{m}\right)$.

The value of $\lambda_{\max }$ has been found to range over $0.35-0.9 \mu \mathrm{m}$, and is typically near $0.55 \mu \mathrm{m}$, which with grain model calculations indicates a 'radius' near $0.1 \mu \mathrm{m}$ for the short dimension of the aligned grains (this depends in detail on composition, shape, and alignment). If there is an underlying power-law size distribution, then some explanation must be sought for why smaller particles of the same composition, which contribute to extinction in the ultraviolet, are not aligned (e.g. Mathis, 1986). The continued decrease of $p(\lambda)$ through the near infrared argues against major contributions from highly absorbing materials (e.g. iron, graphite, glassy carbon, perhaps magnetite), though the merits of some contribution from any specific candidate should be evaluated explicitly. Calculations predict a broadening of $p(\lambda)$ as $m \rightarrow 1$, so that from the observed finite width of $p(\lambda)$ there is some constraint, particularly for prolate particles, on the composition and/or how 'fluffy' aggregated particles might be.

Empirically it appears that the extinction curve is invariant for $\lambda \gtrsim 0.7 \mu \mathrm{m}$ with systematic divergence at shorter wavelengths (e.g. Mathis 1986, Cardelli, Clayton and Mathis, 1988; Whittet, 1988). I have noticed an intriguingly similar behaviour 


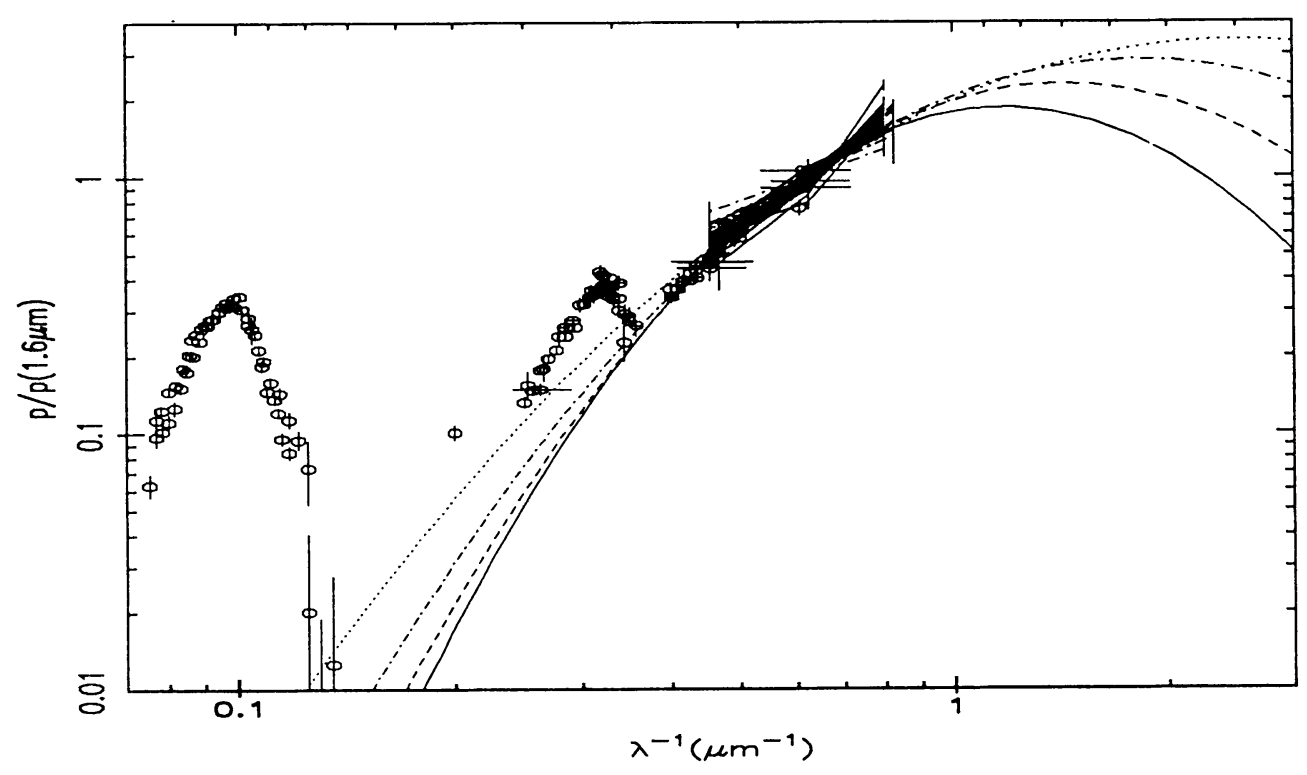

Fig. 1. Composite wavelength dependence of linear polarization. Smooth curves, from a renormalized form of equation 1 with $\lambda_{\max }=0.85,0.7,0.55$ and $0.4 \mu \mathrm{m}$ and $K=-0.10+1.86 \lambda_{\max }$, are similar in the near infrared where data hint at a power-law behaviour. Ice and silicate features for $\mathrm{BN}$ in OMC-1 ( $\lambda_{\max }$ is probably large).

for $p(\lambda)$, which is readily apparent when $p(\lambda)$ is displayed with an unnormalized wavelength scale as in Figure 1 . The near-infrared data shown do not appear to have the same curvature as the smooth curves and there is conceivably a common power-law behaviour with frequency having index $\simeq 2$, not unlike the value 1.7 for extinction. The relative wavelength dependence of polarization and extinction provides an interesting constraint on unified models. By adopting 'invariance' in the near infrared (from 1.2 to $2.2 \mu \mathrm{m}$, say) and yet requiring (perhaps unnecessarily) consistency with the form of equation 1 , I derived the alternative form $K \simeq 1 /[0.5-$ $\left.\ln \left(\lambda_{\max }\right)\right]$.

Larger values of $\lambda_{\max }$ are found in the outer regions of dense clouds. Variations in $\lambda_{\max }$ are correlated with those in the extinction curve (Clayton and Mathis, 1988); for example, $\lambda_{\max } \propto R$, the ratio of total-to-selective extinction. Such a correlation shows that the aligned and any unaligned grain populations respond similarly to environmental changes. The response indicated is that the mean grain size increases in the denser clouds, perhaps the result of the evolution of the size distribution through coagulation. For an assumed composition it should be possible to derive from $p(\lambda)$ objective information on the (evolution of the) size, shape, and orientation distributions using quadratic programming or maximum entropy techniques (Martin, 1978).

For applications over a large wavelength range, Clarke (1984) has questioned the appropriateness of using the form of equation 1 (which embodies a remarkable 
symmetry with respect to $\lambda_{\max }$; see Fig. 2). Systematic deviations might be investigated with spectropolarimetry of higher resolution and precision. Improvement of linear polarization standards is described by Hsu and Breger (1982) and Dolan and Tapia (1976). As a matter of general strategy — and particularly to reveal in $p(\lambda)$ any signature of the wavelength dependence of $m$ which must be present given a sufficiently extended wavelength range - grain model calculations should be compared with actual data, rather than with any smoothed or mean curve on a normalized wavelength scale. Certainly it would be unwise to assume that the form of equation 1 is valid for extrapolation. On the basis of the near-infrared stellar data I have raised the possibility of an alternative power-law dependence. Furthermore, the continuum $p(\lambda)$ for lines of sight in several molecular clouds (e.g. BN in OMC-1; see Fig. 1), while not necessarily the prototype for polarization in diffuse clouds (particularly if confused by scattering!), does appear to be flatter than equation 1 (with large $\lambda_{\max }$ ) would predict, pointing out the importance of further polarization measurements at 3-5 $\mu \mathrm{m}$ in the diffuse interstellar medium. Similarly, measurements in the ultraviolet from HST and WUPPE are awaited with interest.

\section{OPTICAL CONTINUUM BIREFRINGENCE}

As discussed by Shapiro (1975), the linear birefringence is uniquely connected to the linear dichroism at all frequencies by a Kramers-Kronig integral relation (KKIR). If the polarization is symmetrical in $\ln \left(\lambda_{\max } / \lambda\right)$, then $\lambda_{c}=\lambda_{\max }$; for example, the birefringence corresponding to the particular form of equation 1 is shown in Figure 2 (Martin, 1975a). Given the observed behaviour of linear polarization, the observed characteristic cross-over in circular polarization is not unexpected (Kemp and Wolstencroft, 1972). Martin and Angel (1976) have shown that statistically $\lambda_{c}$ is close to $\lambda_{\max }$, and that $\lambda_{c}$ in fact changes systematically with $\lambda_{\max }$ as expected. Anomalous behaviour in HD 183143 is summarized by Michalsky and Schuster (1977). The sign of the birefringence has been checked as well using measurements toward the Crab Nebula (Martin and Angel, 1984).

How is this connected to grain model calculations? Clearly, if it were possible to adjust (via size, shape, and orientation distributions) the linear polarization of different models to be identical, then by the KKIR there would be no independent diagnostic difference to be obtained from birefringence, say $\lambda_{c} / \lambda_{\max }$. Conversely, if this is not possible, as must be the case at some level, then the circular polarization would also be different; however, it should be possible to confirm any putative diagnostic difference based on circular polarization directly in the linear polarization, given sufficient precision and wavelength coverage (Martin, 1975b). This interrelationship is illustrated in Figure 2 by introducing an asymmetrical wavelength dependence in the parameter $K$ in equation 1 ; by design, this produces only a subtle change in linear polarization near $\lambda_{\max }$, but a potentially diagnostic shift in $\lambda_{c}$. The magnitude of the uncertainty in $\lambda_{c}$ resulting from a $50 \%$ uncertainty in the integral contributions outside the 'optical' range $(\simeq 1-3 \mu \mathrm{m}$ for which equation 1 was originally developed) is also about that shown by the broken curves in Figure 2. These illustrations demonstrate a certain localization in the KKIR, so that only the effects of gross deviations in the linear polarization in the infrared and ultraviolet would be detected easily. There is also a practical problem of using the relationship of $\lambda_{\max }$ to $\lambda_{c}$ as a precise constraint on grain models; if the symmetrical form of equation 1 is assumed, then from only limited data sampled around the peak of an underlying asymmetrical curve the apparent $\lambda_{\max }$ recovered would be shifted closer 


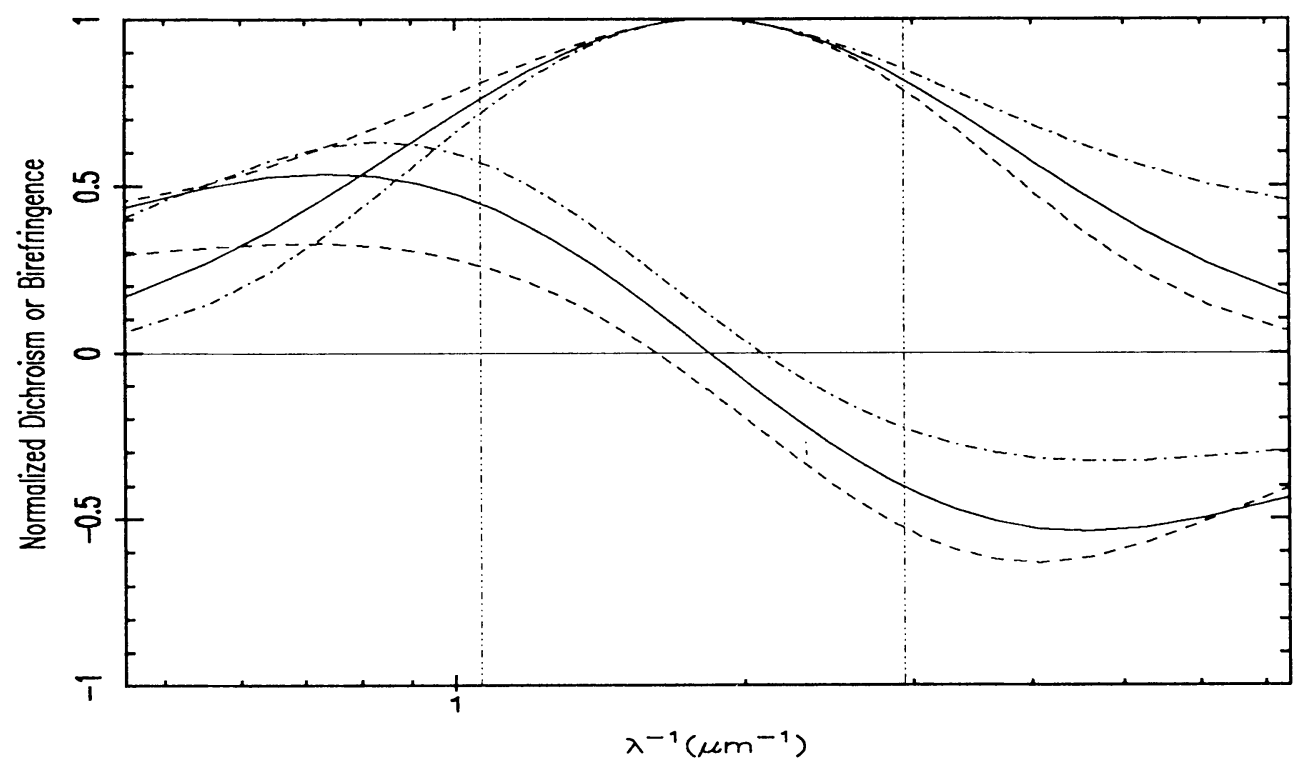

Fig. 2. Birefringence derived from dichroism by a Kramers-Kronig relation. The upper solid curve is the normalized linear dichroism from equation 1 with $\lambda_{\max }=0.545 \mu \mathrm{m}$ and $K=0.91$. The solid curve passing through zero at $\lambda_{\max }$ is the derived linear birefringence. Broken curves indicate how an asymmetry (relative to $\lambda_{\max }$ ) in the linear dichroism affects the derived linear birefringence. Vertical dash-dot lines mark the 'optical' range. Any constraint based on $\lambda_{c} / \lambda_{\max }$ could be obtained directly by measuring $p(\lambda)$ over a large wavelength range.

to $\lambda_{c}$. Obviously direct measurements of linear polarization in the infrared and ultraviolet, which would reveal more subtle changes and spectral signatures, are to be preferred.

On the other hand, $n$ and $k$, on which the relative behaviour of linear dichroism and birefringence in the model depend, are themselves related by a Kramers-Kronig integral relation, and so consistent calculations can be carried out even over a relatively narrow wavelength range. This was my motivation for recommending models and measurements of dichroism and birefringence as a possible discriminant among grain models (Martin, 1972, 1975b). A critical requirement is of course that $n(\lambda)$ and $k(\lambda)$ used in the calculations satisfy the Kramers-Kronig relation. In particular it is important to note that the rough characterization $n \simeq k \simeq$ constant for 'metals' in Martin (1972) does not hold in general over a very extended wavelength range (Martin, 1975b; Chlewicki and Greenberg, 1989a). The actual strong wavelength dependence of $m$ for such materials can make it difficult to reproduce even the linear polarization, especially now that observational coverage has been extended into the near infrared. This failure might be signalled by a diagnostic shift of $\lambda_{c}$ relative to $\lambda_{\max }$, but if the model polarization is so poorly fit by equation 1 , then the evaluation of $\lambda_{\max }$ in practice is problematical (see the example of magnetite in Martin, 1975a). 
At the other extreme, it seems that models incorporating materials that are not strongly absorbing, like silicates, can explain both the continuum linear and circular polarization. Furthermore, the observed polarization structure at the 10 and $19-\mu \mathrm{m}$ silicate features (see Fig. 1 and $\S 5$ ) now reveals directly that silicate grains (at least) can be aligned. Because silicates have an albedo in the optical higher than that of the grain ensemble in the interstellar medium, at least two grain populations (or grain mantles) are required, but the alignment could differ. Polarization measurements at the silicate features in the diffuse interstellar medium where the accompanying continuum optical polarization is well known would be valuable in evaluating whether silicate polarization alone is sufficient.

An interesting related issue is whether any constraint can be placed on mildly absorbing materials (organic refractories, dirty silicates). Using constant refractive indices, which is quite a good approximation for such materials over the observed range, I found a tendency for $\lambda_{c}$ to increase relative to $\lambda_{\max }$ as the absorption increased (Martin, 1974, 1975a); the sensitivity to $k$ depended on $n-1$, suggesting a quantification in terms of something like the albedo, rather than simply $k$ without reference to $n$. However, the tight constraints cited by Mathis (1983) seem at present to be too optimistic, because of uncertainties in the observations themselves, and in their parameterization. As an illustration of the latter point, note that empirically the derived $\lambda_{\max }$ (to be compared to $\lambda_{c}$ ) depends on whether near-infrared data are included in the fit and on the functional form assumed for $p(\lambda)$, particularly when $\lambda_{\max }<0.5 \mu \mathrm{m}$.

In the case of small but finite $k$, often taken to extend into the near-infrared, the diagnostic complementary to increasing $\lambda_{c} / \lambda_{\max }$ is a slower decrease in linear polarization toward low frequencies, qualitatively like the deviation shown in the dashed curve in Figure 2 (in the limit the power-law index in this range would approach 1 rather than the observed 2). For example, the predicted linear polarization of the organic refractory model, as presented in Chlewicki and Greenberg (1989b), passes above the near-infrared measurements (note that there are none beyond $2.2 \mu \mathrm{m}$ ), and $\lambda_{c}$ is slightly larger than $\lambda_{m a x}$; given present data, the near-infrared linear polarization is probably the more serious problem to be addressed. A dirty silicate model with qualitatively similar behaviour is shown in Martin (1974).

One can look forward to extensive ultraviolet to infrared linear polarization measurements which would allow a more precise prediction of the birefringence. At present, it still seems prudent to test a grain model, which contains many adjustable parameters $(\S 2)$, against both linear and circular polarization. Ideally, the model should be compared to actual data (e. g. Martin, 1974) for many stars with different $\lambda_{\max }$, rather than to normalized (and smoothed) fits to the data or to general requirements like $\lambda_{\max } \simeq \lambda_{c}$. This would alleviate uncertainties relating to the appropriateness of the fitting functions and the extraction of $\lambda_{\text {max }}$; it would also be clearer which are the most critical data in evaluating a model, or what new data would be most desirable.

\section{POLARIZATION ACROSS SPECTRAL FEATURES}

One of the most specific indicators of the composition of interstellar grains is the spectral feature at $10 \mu \mathrm{m}$ attributed to silicates. The feature is seen against background/embedded sources not only in extinction but also in linear polarization, 
consistent with earlier independent fits of the continuum polarization by such dielectric particles. An important issue for which $10-\mu \mathrm{m}$ polarization might offer some resolution is the actual form in which silicate materials are found in interstellar grains: bare particles (Mathis, Rumpl, and Nordsieck, 1977), possibly with icy mantles in dark molecular cloud environments (e.g. Lee and Draine, 1985); silicate cores with substantial organic refractory mantles (Greenberg, van de Bult, and Allamandola, 1983; Tielens and Allamandola, 1987; Chlewicki and Greenberg, 1989a); or silicate cores coated with hydrogenated amorphous carbon (HAC; Duley, 1987; Duley, Jones, and Williams, 1989).

For an intrinsically weak resonance in a particle small relative to the wavelength, the wavelength dependence of the cross-section reflects the peaking of the imaginary part of $\epsilon$, and the polarization and extinction profiles are the same. With growing importance for stronger resonances (larger band strengths), the profile responds to anomalous dispersion in the real part of $\epsilon$. Dependencies on shape and orientation of the $E$-vector are introduced through the 'depolarization factor', so that the polarization (linear dichroism) profile, which is the difference between two principal cross-sections ( $(2)$, changes in shape and appears shifted, for either elongated or flattened particles, toward longer wavelengths relative to the extinction profile, which is the sum of the two cross-sections. These effects become apparent for band strengths typical of amorphous silicates, and appear to be relevant for interstellar grains since the polarization profile observed (e.g. Fig. 1) is displaced relative to the absorption profile (Martin, 1975c; Aitken et al., 1985).

When a silicate core is coated with a mantle, similar effects on the profile occur, but because terms like $\left(\epsilon_{\text {core }}-\epsilon_{\text {mantle }}\right)$ rather than $\left(\epsilon_{\text {core }}-1\right)$ appear in the polarizability, the effects relating to anomalous dispersion are more pronounced for a given band strength. Consequently, important yet uncertain parameters which appear in grain models - mantle size and mantle composition, together with the opacity of the silicate material at $10 \mu \mathrm{m}$ - might be constrained by the polarization observations.

From an extensive, though not exhaustive, exploration of parameter space, I believe that Figure 3 provides a fair indication of the merits of this approach. The adopted core is the 'astronomical silicate' of Draine and Lee (1984), with central band strength $\simeq 10^{4} \mathrm{~cm}^{-1}$. Bare oblate particles (here with axial ratio 2:1) are preferred because the relative shift is less pronounced than for bare prolate particles (not shown). Ice and organic refractory (or perhaps HAC) mantles are crudely represented by $m=1.4-0.02 i$ and $2-0.1 i$, respectively. A mantle volume equal to the core volume is assumed. Even so the mantle-induced change in the polarization profile is significant and probably too radical to accommodate the data, particularly for the organic refractory because it has a higher $n$ (note also the polarization reversal). The shift can be diminished by reducing the band strength; however, there is not much leeway there because the strength of the astronomical feature must be reproduced by a silicate mass limited by the cosmic abundance of $S i$ (Lee and Draine, 1985). The relatively small shift observed appears to be a strong challenge to at least those organic refractory models in which the ratio of mantle volume to core volume is large (values up to 10 have often been cited).

If the material producing the $3.1-\mu \mathrm{m}$ ice feature in embedded sources such as $\mathrm{BN}$ is a coating on the aligned silicate grains, then there should be polarization structure at this feature too (Martin, 1975c). This has been found in BN (see 


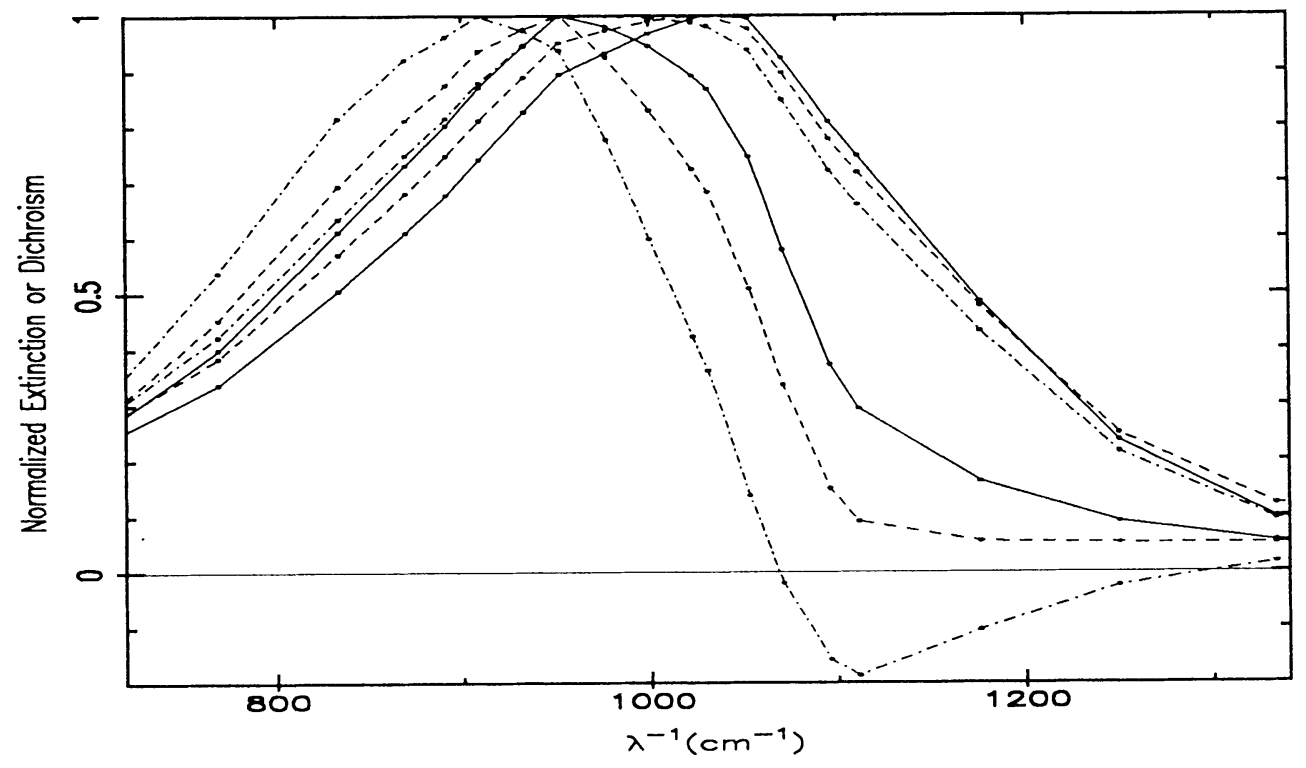

Fig. 3. Comparison of the silicate $10-\mu \mathrm{m}$ linear polarization profile with the extinction profile (curves overlapping on the right). Curves are solid for bare 2:1 oblate cores, and dashed and dot-dashed for cores with 'ice' and 'organic refractory' mantles of equal volume, respectively.

Figure 1), including the expected displacement of the polarization feature relative to the extinction feature (Kobayashi et al., 1980). Circular polarization structure can be modelled too (e.g. Lee and Draine, 1985).

It seems unlikely that graphite makes a predominant contribution to optical continuum polarization. Nevertheless, aligned graphite particles have often been proposed as among the components producing the infrared continuum polarization in molecular cloud sources (e.g. Lee and Draine, 1985). If the $2200 \AA$ feature were produced by aligned graphite particles, there would be interesting polarization structure diagnostic of the shape and anisotropy of the particles (Draine, 1988). Two observations suggestive of little 'excess' polarization (Gehrels, 1974b) should not dampen enthusiasm for the more extensive measurements anticipated from HST and WUPPE.

Sensitive observations across three diffuse interstellar bands (Martin and Angel, 1975) do not show a linear polarization signature as would be expected if the bands originated in aligned grains.

Small amplitude very broad structure (on a scale of $200 \AA$ with relative amplitude $\simeq 1 \%$ and with commensurate swings in position angle), gauged relative to the smooth Serkowski curve, has been identified in a few stars (Wolstencroft and Smith, 1984). However, the structure is not the same from star to star, nor has it been established to be closely related to very broad structure in the extinction curve, and so the precise interpretation is not yet clear. 


\section{INTERSTELLAR POLARIZATION IN OTHER GALAXIES}

Exploration of interstellar polarization and extinction produced in the different environments of other galaxies might enhance our understanding of key issues such as grain composition and alignment. For a discussion of extinction see Fitzpatrick (1989). So far polarimetric studies are not highly developed, though there is surely the potential for more refined observations. Equation 1 has been used to quantify $\lambda_{\text {max }}$; however, except for Cen A (NGC 5128) the data are of insufficient quality to judge the appropriateness of this form.

Observation of a single globular cluster S78 in M31 suggests $\lambda_{\max } \simeq 0.4 \mu \mathrm{m}$ (Martin and Shawl, 1982). Data for several stars: in the LMC (Clayton, Martin, and Thompson, 1983) indicate a range in $\lambda_{\max }$, and some possible deviations from equation 1. Similar results for three stars in the SMC were presented by Magalhães et al., 1989. Polarimetric observations, over the range $0.36-1.64 \mu \mathrm{m}$ of a supernova located behind the dust lane in Cen A (Hough et al., 1987) are described well by equation $1\left(\lambda_{\max }=0.43 \mu \mathrm{m}\right.$ with $\left.K=1.15\right)$.

It will be important to understand the remarkable fact that these $\lambda_{\max }$ values are apparently so similar to those in the Galaxy, given the many factors like composition, size, shape, and alignment at play. Furthermore, polarization to extinction ratios are not unlike the Galactic range and the Galactic relationship of $\lambda_{\max }$ to $R$ (Section 3.) is not contradicted either.

Polarization of the emission lines and continuum in the nucleus of the Seyfert galaxy IC 4329A might be of interstellar origin (Martin et al., 1982), in which case the steep rise toward short wavelengths indicates unprecedentedly small (by a factor three) aligned grains. Acquisition of better spectropolarimetric data for this and other potentially similar galaxies (Thompson and Martin, 1988) might be rewarding.

ACKNOWLEDGEMENTS. This work was supported by the Natural Sciences and Engineering Research Council of Canada.

\section{REFERENCES}

Aitken, D. K., Bailey, J. E., Roche, P. F., and Hough, J. H. 1985, M. N. R. A. S., $215,815$.

Aitken, D. K. 1989, in IAU Symposium 195, Interstellar Dust, eds. L. J. Allamandola and A. G. G. M. Tielens, (Dordrecht: Kluwer), p. 47.

Bohren, C. F., and Huffman, D. R. 1983, Absorption and Scattering of Light by Small Particles, (New York: Wiley).

Cardelli, J. A., Clayton, G. C., and Mathis, J. S. 1988, preprint.

Chlewicki, G., and Greenberg, J. M. 1989a, Ap. J., in press.

C. 1989 b, in Interstellar Dust Contributed Papers, eds. A. G. G. M. Tielens and L. J. Allamandola, NASA CP-3036.

Clarke, D. 1984, M. N. R. A. S., 206, 739.

Clayton, G. C., Martin, P. G., and Thompson, I. 1983, Ap. J., 265, 194.

Clayton, G. C., and Mathis, J. S. 1988, Ap. J., in press.

Cugnon, P. 1989, in Interstellar Dust Contributed Papers, eds. A. G. G. M. Tielens and L. J. Allamandola, NASA CP-3036.

Dolan, J. F., and Tapia, S. 1976, Pub. A. S. P., 98, 792. 
Draine, B. T. 1988, preprint.

Draine, B. T., and Lee, H. M. 1984, Ap. J., 285, 89.

Duley, W. W. 1987, M. N. R. A. S., 229, 203.

Duley, W. W., Jones, A. P., and Williams, D. A. 1989, M. N. R. A. S., in press.

Fitzpatrick, E. 1989, in IAU Symposium 195, Interstellar Dust, eds. L. J. Allamandola and A. G. G. M. Tielens, (Dordrecht: Kluwer), p. 37.

Gehrels, T. 1974a, in Planets, Stars, and Nebulae Studied with Photopolarimetry, ed. T. Gehrels (Tucson: Univ. Arisona Press), p. 52. $19746, A . J ., 79,590$.

Gilra, D. P. 1972, in The Scientific Results of OAO-2, ed. A. D. Code, (NASA SP-310), p. 295.

Greenberg, J. M., van de Bult, C. E. P. M., and Allamandola, L. J. 1983, J. Phys. Chem., 87, 4243.

Hildebrand, R. H. 1988, Quart. J. R. A. S., in press.

Hildebrand, R. H. 1989, in IAU Symposium 1S5, Interstellar Dust, eds. L. J. Allamandola and A. G. G. M. Tielens, (Dordrecht: Kluwer), p. 275.

Hough, J. H., Bailey, J. E., Rouse, M. F., and Whittet, D. C. B. 1987, M. N. R. A. S., 227, 1 P.

Hsu, J. C., and Breger, M. 1982, Ap. J., 262, 732.

Kemp, J. C., and Wolstencroft, R. D. 1972, Ap. J. (Letters), 176, L115.

Kobayashi, Y., Kawara, K., Sato, S., and Okuda, H. 1980, Pub. Astr. Soc. Japan, 82, 295.

Lee, H. M., and Draine, B. T. 1985, Ap. J., 290, 211.

Magalhães, A. M., Piirola, V., Coyne, G. V., Rodrigues, C. V. 1989, in Interstellar Dust Contributed Papers, eds. A. G. G. M. Tielens and L. J. Allamandola, NASA CP-3036.

Martin, P. G. 1972, M. N. R. A. S., 159, 179.

- . 1974, Ap. J., 187, 461.

-. $1975 a$, Ap. J., $201,373$.

- $1975 b, A$. J., $202,389$.

- 1975 c, Ap. J., 202, 393.

. 1978, in Cosmic Dust, (Oxford: Oxford University Press).

Martin, P. G., and Angel, J. R. P. 1974, Ap. J., 193, 343.

- . 1975, Ap. J., 195, 379.

- 1976, Ap. J., 207, 126.

Martin, P. G., and Shawl, S. J. 1982, Ap. J., $258,86$.

Martin, P. G., Stockman, H. S., Angel, J. R. P., Masa, J., and Beaver, E. A. 1982, Ap. J., 255, 65.

Mathis, J. S. 1983, Ap. J., 267, 119.

- 1986, Ap. J., 308, 281.

Mathis, J. S., Rumpl, W., and Nordsieck, K. H. 1977 , Ap. J., 217, 425.

Matsumura, M. and Seki, M. 1989, in Interstellar Dust Contributed Papers, ods. A. G. G. M. Tielens and L. J. Allamandola, NASA CP-3036.

Michalsky, J. J., and Schuster, G. J. 1977, Ap. J., $281,73$.

Onaka, T. 1980, Ann. Tokyo Astron. Obs, end Ser., $18,1$.

Seki, M., and Hasegawa, T. I. 1986, Science Reports of the Tôhoku Univ., 8th Ser., T, 135.

Shah, G. A. $1970, M$. N. R. A. S., 148, 93.

Shapiro, P. R. 1975, Ap. J., 201, 151.

Tielens, A. G. G. M., and Ällamandola, L. J. 1987, in Interstellar Processes, eds. D. J. Hollenbach and H. A. Thronson, (Dordrecht: Reidel), p. 397.

Thompson, I. B., and Martin, P. G. 1988, Ap. J., 380, 121.

Wilking, B. A., Lebofsky, M. J., and Rieke, G. H. 1982, Ap. J., 87, 695.

Whittet, D. C. B. 1988, in Dust in the Universe, eds. M. E. Bailey and D. A. Williams, (Camibridge: Cambridge Univ. Press), in press.

Wolstencroft, R. D., and Smith, R. J. 1984, Occ. Rep. R. Obs. Edinb., $12,167$. 


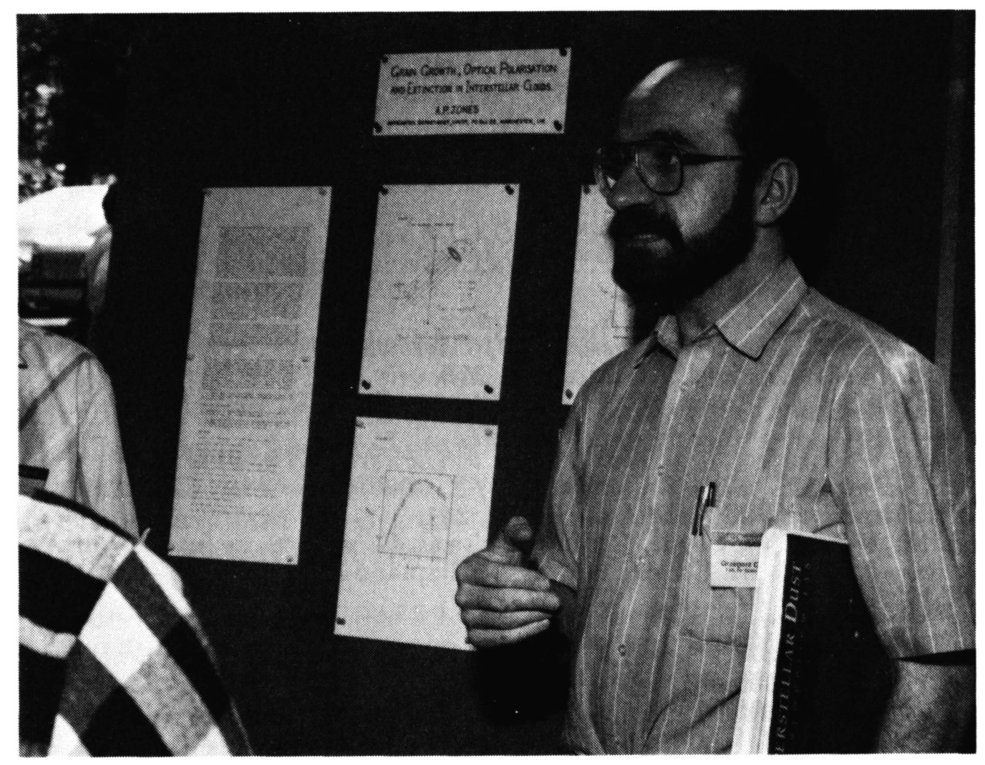

Grzegorz Chlewicki 